\title{
Interval Linear Assignment Problems
}

\author{
Sarangam Majumdar \\ Department of Mathematics, National Institute of Technology, Rourkela, Odisha -769008, India \\ *Corresponding Author: majumdarsarangam@yahoo.in
}

Copyright (C) 2013 Horizon Research Publishing All rights reserved.

\begin{abstract}
Linear assignment problems are very well known linear programming problems. In a ground reality the entries of the cost matrix is not always crisp. In many application this parameters are uncertain and this uncertain parameters are represented by interval. In this contribution we propose interval Hungarian method and consider interval analysis concept for solving interval linear assignment problems.
\end{abstract}

Keywords Interval Analysis, Assignment Problems, Crisp

\section{Introduction}

In practical field we are sometime faced with type of problem which consists of jobs to machines, drivers to trucks, men to offices etc. in which the assignees possess varying degree of efficiency, called as cost or effectiveness .The basic assumption of this type of problem is that one person can perform one job at a time. An assignment plan is optimal if it minimizes the total cost or maximizes the profit. This type of linear assignment problems can be solved by the very well-known Hungarian method which was derived by the two mathematician D. Kö nig and E.Egerváry [11]. Authors are proposed different methods to handle different types of assignment problems. In this context, Albrecher $[1,10]$ introduced an asymptotic behaviour of bottleneck problems and Aldous [2] studied asymptotes in the random assignment problem. Alvis and Lai [3] coined out the probabilistic analysis of a heuristic for the assignment problem .Quadratic assignment was analyzed by many authors in different approaches [4, 5, 7, 8]. Burkard [6] proposed time-slot assignment for TDMA systems .Burkard et al. [9] studied an algebraic approach to assignment problems. Pardalos and Pitsoulis [12] developed some works on nonlinear assignment problems. Asymptotic properties of the random assignment problem have been studied by Olin [15]. Nair Proved of the Parisi and Coppersmith-Sorkin conjectures in the random assignment problem [16]. Fitness landscape analysis and memetic algorithms for the quadratic assignment problem are described by Merz and Freisleben [17]. Optimal
Permutations and Bottleneck quadratic assignment problems and the bandwidth problem were analyzed by $\mathrm{Li}$ et al. and Kellerer et al. [18, 20]. Krokhmal et al. [19] described asymptotic behavior of the expected optimal value of the multidimensional assignment problem.

In the realistic problems costs are not always in a crisp form, sometime these parameters are uncertain which are represented by intervals. Hence we need the help of interval analysis for handling this type of data. In this paper a general interval linear assignment problem is taken into consideration with basis assumption that one person can perform one job at a time. Here the new method has been proposed to handle such type of problem. We solved one example problem using this proposed method. Corresponding results are computed and has been reported here . The rest of the paper is organized as follows: in the next section review on interval arithmetic are highlighted. In section 3 the detail of proposed interval Hungarian method are presented. Example problem is solved and result is analyzed in section 4.Finally conclusions are drawn in section 5 .

\section{Interval Arithmetic}

The interval form of the parameters may be written as

$$
[x, \bar{x}]=\{x: x \in \mathfrak{R}, \underline{x} \leq x \leq \bar{x}\}
$$

where $\underline{x}$ is the left value and $\bar{x}$ is the right value of the interval respectively. We define $m=\frac{x+\bar{x}}{2}$ is the centre and $w=\bar{x}-\underline{x}$ is the width of the interval $[x, \bar{x}]$.

Let $[\underline{x}, \bar{x}]$ and $[\underline{y}, \bar{y}]$ be two elements then the following arithmetic are well known [14]
(i) $[\underline{x}, \bar{x}]+[\underline{y}, \bar{y}]=[\underline{x}+\underline{y}, \bar{x}+\bar{y}]$
(ii) $[\underline{x}, \bar{x}]-[\underline{y}, \bar{y}]=[\underline{x}-\bar{y}, \bar{x}-\underline{y}]$
(iii) $[\underline{x}, \bar{x}] \times[y, \bar{y}]=[\min \{\underline{x} y, \underline{x} \bar{y}, \bar{x} y, \overline{x y}\}$, $\max \{\underline{x} \underline{y}, \underline{x} \bar{y}, \bar{x} \underline{y}, \overline{x y}\}]$
(iv) $[\underline{x}, \bar{x}] \div[\underline{y}, \bar{y}]=[\min \{\underline{x} \div \underline{y}, \underline{x} \div \bar{y}, \bar{x} \div \underline{y}, \bar{x} \div \bar{y}\}$, 
$\max \{\underline{x} \div \underline{y}, \underline{x} \div \bar{y}, \bar{x} \div \underline{y}, \bar{x} \div \bar{y}\}]$ provide if $[\underline{y}, \bar{y}] \neq[0$, 0]

\section{Interval Hungarian Method}

In this section we are proposed interval Hungarian method to solve the interval linear assignment problems.

Algorithm:

Step 1 Find out the mid values of each interval in the cost matrix.

Step 2 Subtract the interval which have smallest mid value in each row from all the entries of its row.

Step 3 Subtract the interval which have smallest mid value from those columns which have no intervals contain zero from all the entries of its column.

Step 4 Draw lines through appropriate rows and columns so that all the intervals contain zero of the cost matrix are covered and the minimum number of such lines is used.

Step 5 Test for optimality (i) If the minimum number of covering lines is equal to the order of the cost matrix, then optimality is reached. (ii) If the minimum number of covering lines is less than the order of the matrix, then go to step 6.

Step 6 Determine the smallest mid value of the intervals which are not covered by any lines .Subtract this entry from all un-crossed element elements and add it to the crossing having an interval contain zero. Then go to step 4 .

\section{Numerical Results}

We take a linear assignment problem as an example problem and solved this problem by traditional Hungarian method .The assignment cost of assigning any operator to any one machine is given in the following table [13].

Table 1. Cost matrix with crisp entries

\begin{tabular}{|c|c|c|c|c|}
\hline & I & II & III & IV \\
\hline A & 10 & 5 & 13 & 15 \\
\hline B & 3 & 9 & 18 & 3 \\
\hline C & 10 & 7 & 3 & 2 \\
\hline D & 5 & 11 & 9 & 7 \\
\hline
\end{tabular}

Now we are applying Hungarian method, then we get an optimal assignment as A,B,C,D machines are assign to II , IV ,III,I operators respectively and minimum assignment cost is 16 .

Now change the entry of the cost matrix by some interval form. Then we get a new cost matrix as follow.

Table 2. Cost matrix with interval entries

\begin{tabular}{|c|c|c|c|c|}
\hline & I & II & III & IV \\
\hline A & {$[9,11]$} & {$[4,6]$} & {$[12,14]$} & {$[14,16]$} \\
\hline B & {$[2,4]$} & {$[8,10]$} & {$[17,19]$} & {$[2,4]$} \\
\hline C & {$[9,11]$} & {$[6,8]$} & {$[2,4]$} & {$[1,3]$} \\
\hline D & {$[4,6]$} & {$[10,12]$} & {$[8,10]$} & {$[6,8]$} \\
\hline
\end{tabular}

We are applying the proposed interval Hungarian method and solve this problem. We get an minimum assignment cost is $[14,22]$ and optimal assignment as A ,B,C,D machines are assign to II ,I, III ,IV operators respectively .But this solution is not unique because we can find out another assignment as A , B,C ,D are assign to II, IV ,III,I respectively and minimum assignment cost is [12, 20].

\section{Conclusions}

The proposed interval Hungarian method is effective and useful in this interval context. Using this method we can solve real world linear assignment problems where entries of the cost matrix are interval form. Generalized linear assignment problems can be solved by this proposed method.

\section{Acknowledgements}

Author would like to thanks Prof. Suman Datta, Prof. S. Chakraverty and reviewers for their valuable comments and discussion.

\section{REFERENCES}

[1] Albrecher, H. (2005) "A note on the asymptotic behaviour of bottleneck problems," Operations Research Letters, 33, 183-186

[2] Aldous, D. (1992) "Asymptotics in the random assignment problem," Probability Theory and Related Fields, 93, 507-534.

[3] Alvis, D. and Lai, C.W. (1988) "The probabilistic analysis of a heuristic for the assignment problem," SIAM Journal on Computing, 17, 732-741.

[4] Anstreicher, K. M. (2003) "Recent advances in the solution of quadratic assignment problems," Mathematical Programming, 97 (1-2), 27-42.

[5] Buck, M.W., Chan, C. S., and Robbins, D. P. (2002) "On the Expected Value of the Minimum Assignment," Random Structures \& Algorithms, 21 (1), 33-58.

[6] Burkard, R. E. (1985) "Time-slot assignment for TDMA systems," Computing, 35 (2), 99-112.

[7] Burkard, R. E. and Fincke, U. (1982a) "The Asymptotic Probabilistic Behavior of Quadratic Sum Assignment Problems," Zeitschrift für Operations Research, 27, 73-81.

[8] Burkard, R. E. and Fincke, U. (1982b) "On random quadratic bottleneck assignment problems," Mathematical Programming, 23, 227-232.

[9] Burkard, R. E., Hahn, W., and Zimmermann, U. (1977) “An algebraic approach to assignment problems," Mathematical Programming, 12, 318-327 
[10] Gabow, H. N. and Tarjan, R. E. (1988) "Algorithms for two bottleneck assignment problems," Journal of Algorithms, 9 (3), 411-417

[11] Kuhn, H. W. (1955) "The Hungarian method for the assignment problem," Naval Research Logistics Quarterly, 2 (1-2), 83-87.

[12] Pardalos, P. M. and Pitsoulis, L. (Eds.) (2000) Nonlinear Assignment Problems: Algorithms and Applications, Kluwer Academic Publishers.

[13] Hamdy A. Taha, Operations Research (Eighth Edition)(2008), Pearson, ISBN -9780131889231.

[14] Cloud, Michael J.; Moore, Ramon E.; Kearfott, R. Baker, Introduction to Interval Analysis. Philadelphia: Society for Industrial and Applied Mathematics. ISBN 0-89871-669-1(2009).

[15] Olin, B. (1992) Asymptotic properties of the random assignment problem, PhD thesis, Department of Mathematics, Royal Institute of Technology, Stockholm, Sweden.

[16] Nair, C. (2005) Proofs of the Parisi and Coppersmith-Sorkin conjectures in the random assignment problem, $\mathrm{Ph} . \mathrm{D}$. thesis, Stanford University.

[17] Merz, P. and Freisleben, B. (2000) "Fitness landscape analysis and memetic algorithms for the quadratic assignment problem," IEEE Transactions on Evolutionary Computation, 4 (4), 337-352.

[18] Li, Y. and Pardalos, P. M. (1992) "Generating Quadratic Assignment Test Problems with Known Optimal Permutations," Computational Optimization and Applications, 1 (2), 163-184.

[19] Krokhmal, P., Grundel, D., and Pardalos, P. (2007) "Asymptotic Behavior of the Expected Optimal Value of the Multidimensional Assignment Problem," Mathematical Programming, 109 (2-3), 525-551.

[20] Kellerer, H. and Wirsching, G. (1998) "Bottleneck quadratic assignment problems and the bandwidth problem," Asia-Pacific Journal of Operational Research, 15 (2), 169-177. 